\title{
Acquired cold urticaria: Clinical features, particular phenotypes, and disease course in a tertiary care center cohort
}

\author{
Gustavo Deza, MD, ${ }^{\mathrm{a}}$ Ana Brasileiro, MD, ${ }^{\mathrm{a}, \mathrm{b}}$ Marta Bertolín-Colilla, MD, ${ }^{\mathrm{a}}$ Laia Curto-Barredo, MD, \\ Ramon M. Pujol, MD, PhD, and Ana M. Giménez-Arnau, MD, PhD ${ }^{\mathrm{a}}$ \\ Barcelona, Spain; and Lisbon, Portugal
} Background: Data about special phenotypes, natural course, and prognostic variables of patients with
acquired cold urticaria (ACU) are scarce.

Objectives: We sought to describe the clinical features and disease course of patients with ACU, with special attention paid to particular phenotypes, and to examine possible parameters that could predict the evolution of the disease.

Methods: This study was a retrospective chart review of 74 patients with ACU who visited a tertiary referral center of urticaria between 2005 and 2015.

Results: Fourteen patients (18.9\%) presented with life-threatening reactions after cold exposure, and 21 (28.4\%) showed negative results after cold stimulation tests (classified as atypical ACU). Nineteen patients (25.7\%) achieved complete symptoms resolution at the end of the surveillance period and had no subsequent recurrences. Higher rates of atypical ACU along with a lower likelihood of achieving complete symptom resolution was observed in patients who had an onset of symptoms during childhood $(P<.05)$. In patients with atypical ACU, shorter disease duration and lower doses of antihistamines required for achieving disease control were detected $(P<.05)$. Age at disease onset, symptom severity, and cold urticaria threshold values were found to be related to disease evolution $(P<.05)$.

Limitations: This study was limited by its retrospective nature.

Conclusions: The knowledge of the clinical predictors of the disease evolution along with the clinical features of ACU phenotypes would allow for the establishment of an early and proper therapeutic strategy. ( J Am Acad Dermatol 2016;75:918-24.)

Key words: acquired cold urticaria; disease course; phenotypes; physical urticaria.

A cquired cold urticaria (ACU) represents a form of inducible urticaria that is characterized by the development of wheals, angioedema, or both in response to exposure to cold air, solids, or liquids. ${ }^{1}$ ACU symptoms are usually limited to areas of the skin that have been exposed to cold.

From the Departments of Dermatology, Hospital del Mar- Institut Mar d'Investigacions Mèdiques, a Universitat Autònoma de Barcelona (UAB), Barcelona, and the Hospital S. António dos Capuchos, ${ }^{\text {b }}$ Centro Hospitalar Lisboa Central, Lisbon.

Funding sources: None.

Conflicts of interest: None declared.

Ethical approval was granted by the Clinical Research Ethics Committee of the Hospital del Mar (approval no. 2016/6617/I).

Supplemental figures are available at http://www.jaad.org.

\section{Abbreviations used:}

ACU: acquired cold urticaria

CST: cold stimulation test

CsTT: cold stimulation time threshold

CTT: critical temperature threshold 
However, extensive cold contact may result in generalized symptoms, such as headache, dyspnea, hypotension, and a loss of consciousness. ${ }^{1-3}$

A diagnosis of ACU is typically made in patients with a clinical history of wheals or angioedema after cold exposure; the diagnosis should then be appropriately verified with cold stimulation tests (CSTs), the ice cube test being the traditional standardized method. ${ }^{1,2,4}$ In addition, objective methods, such as the TempTest (Emosystems, Berlin, Germany) - a Peltier elementbased provocation device used for the evaluation of the critical temperature threshold (CTT) and the cold stimulation time threshold (CsTT)-are helpful for assessing ACU severity and monitoring therapy efficacy. ${ }^{5-7}$ There is a small percentage of patients that are classified as having atypical ACU; they have identical clinical manifestations but these cannot be reproduced by standard CSTs. Therefore, the diagnosis of atypical ACU relies largely on the patient's clinical history. 1,8

Although previous studies have shown the primary clinical features of ACU, data about particular phenotypes and prognostic variables of the disease in a large series of patients are currently scarce. The aim of this study was to review the clinical, diagnostic, and therapeutic features along with the evolution of different ACU phenotypes and to examine possible parameters that could predict the disease evolution.

\section{MATERIALS AND METHODS Study design}

This retrospective study was based on the review of clinical charts from patients with primary (idiopathic) ACU who were referred to the Urticaria Clinic of the Department of Dermatology of Hospital del Mar (Barcelona) between January 2005 and December 2015. The diagnosis of cold urticaria was based on a patient's clinical history of wheals, angioedema, or both immediately after cold exposure. Patients with positive evidence of an underlying condition associated with the induction of cold urticaria rash (also known as "secondary" ACU [eg, cryoglobulinemia, cold agglutinins, cryofibrinogenemia, leukocytoclastic vasculitis, drugs, or infectious diseases]) or familiar forms of cold urticaria were excluded from the study.
The local clinical research ethics committee from the Hospital del Mar granted ethical approval for the study.

\section{Data collected}

Following a systematized prospective clinical protocol, data about epidemiologic and clinical features, diagnostic procedures, therapeutic outcomes, and disease course were recorded from each patient through chart review.

Atopy was defined by the presence of $\geq 1$ of the following manifestations: atopic dermatitis, allergic rhinitis, or allergic asthma. Disease severity was categorized into 3 types based on the classification suggested by Wanderer et $\mathrm{al}^{10}$ : type I, localized urticaria or angioedema; type II, generalized urticaria or angioedema without hypotensive or respiratory symptoms; and type III, severe systemic reactions with $\geq 1$ episodes suggestive of hypotension (ie, dizziness, sensation of fainting, disorientation, or shock) or respiratory distress (eg, shortness of breath or wheezing). Cold exposures that triggered symptoms were considered: water, solids, air (including the exposure to cold environmental conditions), and eating frozen/ cold foods.

The diagnosis of ACU was supported by the ice cube challenge test. As currently recommended, our standard protocol consisted of the application of the cold stimulus over the patient's forearm for 5 minutes followed by 10 minutes of rewarming. ${ }^{4}$ The test was considered positive if a coalescent wheal was elicited over the application site. In addition, all patients underwent threshold assessment using TempTest 3.0 at the baseline evaluation. The CsTT and the CTT were defined as the shortest time and the highest temperature, respectively, at which a wheal appears through cold provocation. ${ }^{5}$ At the time of testing, all patients were symptom-free and none had received antihistamines or oral corticosteroids during the 10 days before testing. Subjects without wheal formation after the CSTs (ie, the ice cube test and the TempTest) were diagnosed with atypical ACU.

Treatment was based on the continuous intake of second-generation $\mathrm{H}_{1}$-antihistamines at standard dose. If patients did not perceive symptom 
improvement after 15 to 30 days, antihistamines were gradually increased up to 4 times the licensed dose until a complete control of symptoms was achieved. In refractory cases, alternative treatments were considered. Disease control with a particular treatment was defined as an improvement of the signs and symptoms referred by the patient until they did not cause interferences in daily life (therapeutic objective). Antihistamine dosage was defined as the maximum daily dose of medication required for achieving disease control.

The assessment of ACU evolution at the end of the surveillance period was based on the feeling of the patient or the patient's guardian, and accordingly classified as follows: "resolution," when complete freedom of symptoms was achieved after normally cold exposure in $\geq 1$ winter season without medication; "improvement," if decreased severity or frequency of symptoms were noted without achieving a complete resolution and still in need of treatment; and "no improvement," when the severity and frequency of symptoms remained stable or increased in spite of the correct treatment. Disease duration was defined as the time from symptom onset to the last follow-up visit or until complete symptom resolution. In patients who achieved complete symptom resolution, telephone interviews were performed to know if there were disease recurrences.

\section{Statistical analysis}

Descriptive statistics were performed for each variable. Variables were compared descriptively between the different subgroups of patients with ACU using frequencies and percentages for categorical variables and means and standard deviations for quantitative variables. Categorical variables were compared between groups using chi-squared and Fischer's exact tests, while qualitative variables were compared using the Mann-Whitney $\mathrm{U}$ and Kruskal-Wallis $\mathrm{H}$ tests. All calculated $P$ values $\leq .05$ were considered statistically significant.

\section{RESULTS}

\section{Description of the study population}

During the study period, 1030 patients with chronic urticaria were referred to our department. Seventy-four patients (7.2\%) presented with definite symptoms of ACU and were included in the study. All these patients were diagnosed with idiopathic ACU because no secondary causes were found. The clinical characteristics of the study population are summarized in Table I. Forty-seven patients (63.5\%) were female; the mean age was $38.3 \pm 19.0$ years, while the mean age at symptoms onset was $32.0 \pm 18.8$ years. The mean diagnostic delay was
24.5 months (range, 1-144 months). Twenty-five patients (33.8\%) had a personal history of atopy.

Twenty-five patients (33.8\%) presented with a localized form of ACU (type I), and 49 patients (66.2\%) experienced generalized urticaria. The most frequent cold stimulus that induced symptoms was cold water, which was a trigger in 54 patients (73.0\%), followed by exposure to cold air (58.1\%). Twenty-one patients (28.4\%) had a negative result after the ice cube provocation test. In these patients, no wheal formation was detected with the TempTest; therefore, CTT and CsTT were not measurable. In the remaining 53 patients with a positive ice cube test, the mean CTT was $14.0 \pm 7.2^{\circ} \mathrm{C}$ and the mean CsTT was $2.9 \pm 1.4$ minutes.

Regarding the therapeutic outcomes, 73 patients (98.6\%) were treated with different types of $\mathrm{H}_{1}$ antihistamines, achieving disease control in $62 \mathrm{pa}$ tients (84.9\%). Fifty-two of these 62 patients (83.9\%) achieved disease control with 1 or 2 daily doses of antihistamines (21 and 31 subjects, respectively). The remaining 10 patients (16.1\%) required higher doses. Cyclosporine and omalizumab were prescribed in 3 patients each; clinical improvement was shown in only 1 patient treated with cyclosporine, while all 3 patients (100\%) who were treated with omalizumab achieved disease control.

The mean duration of surveillance was $7.5 \pm 3.8$ years. In 46 patients (62.2\%), disease evolution at the end of the surveillance period was classified as "improvement," whereas 9 patients (12.2\%) showed "no improvement." The remaining 19 patients (25.7\%) showed complete symptom resolution, with a mean disease duration until resolution of 5.1 years (range, 1-24 years). No disease recurrences were recorded in these patients. According to the Kaplan-Meier analysis, it was estimated that the percentage of patients showing complete symptom resolution was $26.6 \%$ during the first 5 years of the disease and $44.5 \%$ during the first 10 years (Supplementary Fig 1; available online at www.jaad.org).

\section{Disease severity}

Severity of symptoms was found to be statistically significant related to the rate of disease control by using antihistamines $(P=.002)$, the antihistamine dosage required $(P<.001)$, disease duration $(P=.03)$, and evolution $(P=.03$; Table II). In addition, the unique exposure significantly related to symptom severity was cold water $(P=.01)$.

\section{Pediatric and adult-onset acquired cold urticaria}

Twenty patients (27.0\%) with an early onset of symptoms ( $\leq 18$ years of age) were classified as having 
Table I. Characteristics of acquired cold urticaria in our study population

\begin{tabular}{|c|c|c|c|c|c|c|c|}
\hline Variables & $\begin{array}{l}\text { All patients } \\
(\mathrm{n}=74)\end{array}$ & $\begin{array}{l}\text { Pediatric-onset } \\
\text { ACU }(\mathbf{n}=20)\end{array}$ & $\begin{array}{c}\text { Adult-onset } \\
\text { ACU }(\mathbf{n}=54)\end{array}$ & $P$ value & $\begin{array}{c}\text { Atypical } \\
\text { ACU }(\mathbf{n}=\mathbf{2 1}) \\
\end{array}$ & $\begin{array}{c}\text { Positive } \\
\text { CST }(\mathbf{n}=53)\end{array}$ & $P$ value \\
\hline \multicolumn{8}{|l|}{ Sex, n (\%) } \\
\hline Female & $47(63.5)$ & $13(65.0)$ & $34(63.0)$ & .87 & $12(57.1)$ & $35(66.0)$ & .47 \\
\hline Male & $27(36.5)$ & $7(35.0)$ & $20(37.0)$ & & $9(42.9)$ & $18(34.0)$ & \\
\hline $\begin{array}{l}\text { Mean age at symptom onset, y } \\
\text { (range) }\end{array}$ & $32.0(1-89)$ & $9.1(1-18)$ & $40.5(19-89)$ & NA & $28.7(1-89)$ & $33.4(1-65)$ & .22 \\
\hline Atopy, n (\%) & $25(33.8)$ & $7(35.0)$ & $18(33.3)$ & .89 & $5(23.8)$ & $20(37.7)$ & .25 \\
\hline \multicolumn{8}{|l|}{ Disease severity, n (\%) } \\
\hline 1 & $25(33.8)$ & $6(30.0)$ & $19(35.2)$ & .70 & $9(42.9)$ & $16(30.2)$ & .14 \\
\hline II & $35(47.3)$ & $9(45.0)$ & $26(48.1)$ & & $11(52.4)$ & $24(45.3)$ & \\
\hline III & $14(18.9)$ & $5(25.0)$ & $9(16.7)$ & & $1(4.8)$ & $13(24.5)$ & \\
\hline \multicolumn{8}{|l|}{ Cold trigger, $\mathrm{n}(\%)$} \\
\hline Water & $54(73.0)$ & $15(75.0)$ & $39(72.2)$ & .81 & $14(66.7)$ & $40(75.5)$ & .44 \\
\hline Solids & $28(37.8)$ & $6(30.0)$ & $22(40.7)$ & .39 & $6(28.6)$ & $22(41.5)$ & .30 \\
\hline Air & $43(58.1)$ & $14(70.0)$ & $29(53.7)$ & .20 & $15(71.4)$ & $28(52.8)$ & .14 \\
\hline Food & $5(6.8)$ & $0(0)$ & $5(9.3)$ & .31 & $0(0)$ & $5(9.4)$ & .31 \\
\hline \multicolumn{8}{|l|}{ Ice cube test, n (\%) } \\
\hline Positive & $53(71.6)$ & $10(50.0)$ & $43(79.6)$ & $.01 *$ & NA & NA & NA \\
\hline Negative & $21(28.4)$ & $10(50.0)$ & $11(20.4)$ & & & & \\
\hline Mean CsTT, min (range) & $2.9(1-5)$ & $3.0(1-5)$ & $2.9(1-5)$ & .99 & NA & $3.0(1-5)$ & NA \\
\hline Mean $\mathrm{CT},{ }^{\circ} \mathrm{C}$ (range) & $14.0(4-26)$ & $11.6(4-26)$ & $14.6(4-26)$ & .21 & NA & $14.0(4-26)$ & NA \\
\hline Disease control with anti- $\mathrm{H}_{1}, \mathrm{n}(\%)$ & $62(83.8)$ & $17(85.0)$ & $45(83.3)$ & .86 & $19(90.5)$ & $43(81.1)$ & .33 \\
\hline Mean anti- $\mathrm{H}_{1}$ daily dosage, (range) ${ }^{\dagger}$ & $2.1(1-4)$ & $2.0(1-4)$ & $2.2(1-4)$ & .63 & $1.4(1-2)$ & $2.4(1-4)$ & $.001 *$ \\
\hline \multicolumn{8}{|l|}{ Disease evolution, n (\%) } \\
\hline Resolution & $19(25.7)$ & $1(5.0)$ & $18(33.3)$ & $.03^{*}$ & $4(19.0)$ & $15(28.3)$ & .31 \\
\hline Improvement & $46(62.2)$ & $16(80.0)$ & $30(55.6)$ & & $16(76.2)$ & $30(56.6)$ & \\
\hline No improvement & $9(12.2)$ & $3(15.0)$ & $6(11.1)$ & & $1(4.8)$ & $8(15.1)$ & \\
\hline Mean disease duration, y (range) & $5.3(1-25)$ & $6.9(1-16)$ & $4.8(1-25)$ & .43 & $4.3(1-11)$ & $5.8(1-25)$ & $.02^{*}$ \\
\hline
\end{tabular}

$A C U$, Acquired cold urticaria; $C S T$, cold stimulation test; anti- $H_{1}$, histamine $\mathrm{H}_{1}$-receptor antagonist; $C s T$, cold stimulation time threshold; $C T T$, critical temperature threshold; $N A$, not applicable.

*Statistically significant.

${ }^{\dagger}$ The standard antiH $\mathrm{H}_{1}$ dose $=1$.

pediatric-onset ACU. The frequency of atypical ACU was higher in this population than in patients with adult-onset ACU $(50.0 \%$ vs $20.4 \%$, respectively; $P=.01)$. No statistically significant differences were found between these 2 groups regarding disease severity, cold urticaria triggers, or rate of disease control using antihistamines. However, different disease evolutions were found, with a lower rate of symptom resolution in the patients with pediatriconset ACU (5\% vs 33.3\%; $P=.03$ ).

\section{Results of the cold stimulation tests}

Twenty-one patients (28.4\%) were classified as having atypical ACU based on the negative CSTs results. Some variables, such as symptom severity, cold urticaria triggers, and disease evolution were found to be similar between patients with atypical and typical ACU. Although the frequency of patients achieving disease control using antihistamines was also similar in both groups (90.5\% vs $81.1 \%$, respectively), the antihistamine dosage required was lower in patients with atypical ACU (the mean daily dose of medication was 1.4 times the licensed dose vs 2.4 times the licensed dose; $P=.001$ ). Moreover, disease duration was shorter in patients with atypical ACU compared with patients with positive CSTs ( 4.3 vs 5.8 years; $P=.02$ ).

\section{Threshold assessment}

CTT and CsTT values measured at the baseline evaluation were found to be statistically significant related to disease severity $(P=.004$ and $P=.009$, respectively; Fig 1$)$. CTT was also related to disease evolution $(P=.03)$. CsTT values were slightly higher in patients with complete symptom resolution at the end of the surveillance period than the "improvement" and "no improvement" groups; however, this trend was not statistically significant $(P=.54$; Supplementary Fig 2; available online at www.jaad.org).

\section{DISCUSSION}

Cold urticaria is a cutaneous disorder that represents a small proportion of chronic urticarias $(7-8 \%$ in our series). ACU can affect subjects from both sexes 
Table II. Disease severity of patients with acquired cold urticaria

\begin{tabular}{|c|c|c|c|c|}
\hline \multirow[b]{2}{*}{ Variables } & \multicolumn{3}{|c|}{ ACU type } & \multirow[b]{2}{*}{$P$ value } \\
\hline & $I(n=25)$ & II $(\mathbf{n}=35)$ & III $(\mathbf{n}=14)$ & \\
\hline Mean age at symptom onset, y (range) & $34.9(2-89)$ & $31.1(1-61)$ & $29.3(1-65)$ & .74 \\
\hline Atopy, n (\%) & $6(24.0)$ & $13(37.1)$ & $6(42.9)$ & .42 \\
\hline \multicolumn{5}{|l|}{ Cold trigger, n (\%) } \\
\hline Water & $13(52.0)$ & $28(80.0)$ & $13(92.9)$ & $.01^{*}$ \\
\hline Solids & $10(40.0)$ & $14(40.0)$ & $4(28.6)$ & .73 \\
\hline Air & $16(64.0)$ & $22(62.9)$ & $5(35.7)$ & .17 \\
\hline Food & $2(8.0)$ & $2(5.7)$ & $1(7.1)$ & .74 \\
\hline Disease control with anti- $\mathrm{H}_{1}, \mathrm{n}(\%)$ & $25(100)$ & $30(85.7)$ & $8(57.1)$ & $.002 *$ \\
\hline Mean anti- $\mathrm{H}_{1}$ daily dosage, ${ }^{\dagger}$ (range) & $1.4(1-2)$ & $2.1(1-4)$ & $3.1(2-4)$ & $<.001^{*}$ \\
\hline \multicolumn{5}{|l|}{ Disease evolution, $\mathrm{n}(\%)$} \\
\hline Resolution & $9(36.0)$ & $9(25.7)$ & $1(7.1)$ & $.03^{*}$ \\
\hline Improvement & $16(64.0)$ & $21(60.0)$ & $9(64.3)$ & \\
\hline No improvement & $0(0)$ & $5(14.3)$ & 4 (28.6) & \\
\hline Mean disease duration, y (range) & $4.2(1-24)$ & $5.6(1-22)$ & $6.6(2-25)$ & $.03^{*}$ \\
\hline
\end{tabular}

$A C U$, Acquired cold urticaria; anti- $H_{1}$, histamine $\mathrm{H}_{1}$-receptor antagonist. *Statistically significant.

${ }^{\dagger}$ The standard antiH $\mathrm{H}_{1}$ dose $=1$.
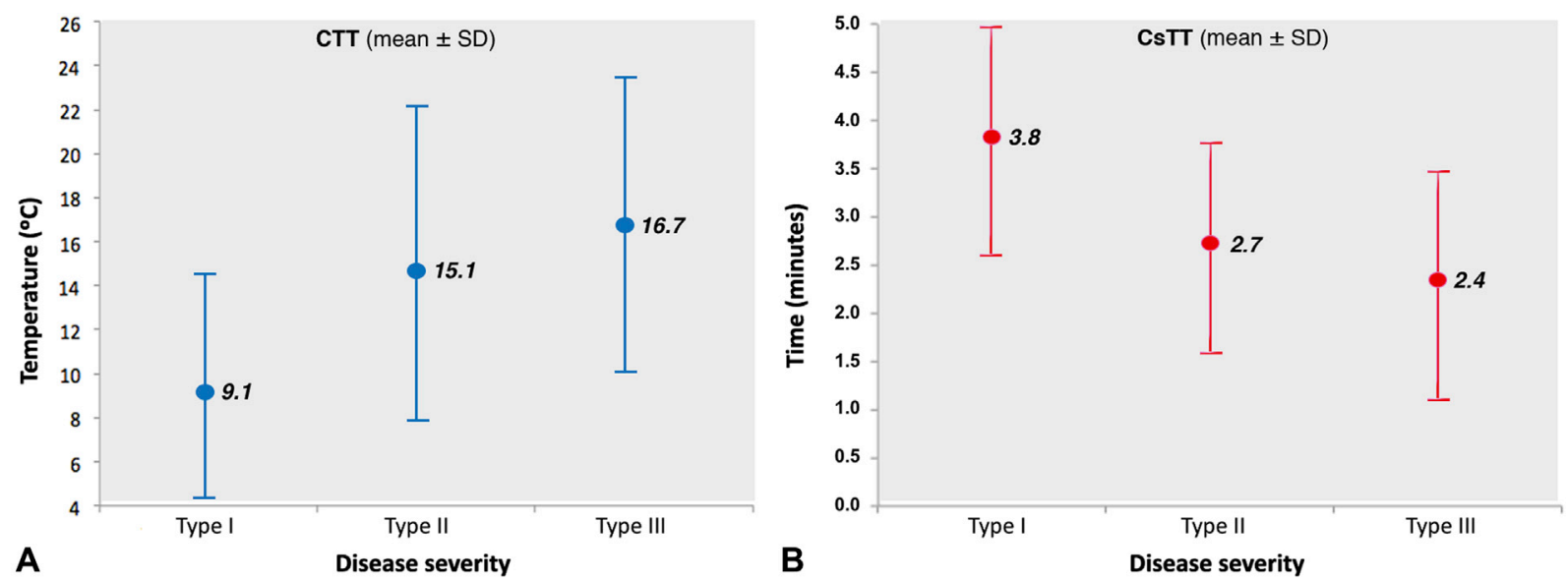

Fig 1. A, Critical temperature threshold (CTT) and (B) cold stimulation time threshold (CsTT) values by categories of disease severity $(P=.004$ and $P=.009$, respectively).

and may start at any age, although it has been postulated that middle-aged women are most often affected. ${ }^{2,5}$ Similarly, we found a female predominance in our population, and the most frequent age at disease onset ranged from 23 to 41 years.

In the present study, disease severity was classified based on the extension of the urticarial reaction and the presence of hypotensive or respiratory symptoms. Accordingly, almost $19 \%$ of patients with ACU can present with life-threatening reactions. The only exposure that was found to be related to disease severity was cold water; $93 \%$ of the patients who suffered systemic reactions were exposed to this stimulus. This can be explained because aquatic activities usually include a wide skin area exposure and involve longer periods of time. Therefore, it seems appropriate for patients with ACU to avoid or minimize the use of aquatic activities. Our results also suggest a relationship between symptom severity and both the disease prognosis and the therapeutic response. Thus, in patients with severe $\mathrm{ACU}$, a longer duration of disease, lower rates of complete symptom resolution, and a poorer therapeutic response to antihistamines would be expected. Therefore, a precise definition of disease severity at the baseline evaluation is recommended, and when severe forms of ACU are detected, a closer follow-up and an early treatment with higher doses of antihistamines seem advisable.

Different phenotypes of patients with ACU were identified. Although clinical features of cold urticaria seem to be similar regardless of the age at symptom 
onset, in our series, patients with pediatric-onset ACU showed some differences; ACU was more difficultly induced through the ice cube test or the TempTest, and a complete resolution of symptoms was less likely to be achieved. These results are consistent with a previous pediatric study ${ }^{3}$ in which complete symptom resolution was achieved in only 2 of 30 (7.4\%) children with cold urticaria, and $41 \%$ of the patients had negative CSTs. Similarly, Santaolalla et $\mathrm{al}^{11}$ noted a full recovery of symptoms in only 1 of 12 pediatric patients analyzed. Therefore, patients with ACU who have an early onset of symptoms and their families should receive counseling regarding the particular prognosis of the disease. Because a longer duration of disease might be expected, these patients should understand their condition and integrate it into their daily lives. Future long-term studies are necessary to validate these data and to fully understand the natural history of ACU in children.

Twenty-eight percent of our patients had negative CSTs and were diagnosed with atypical ACU. The frequency of atypical ACU in previous series varied between $8 \%$ and 49\%. ${ }^{3,9,10,12}$ Although the knowledge of this entity, especially regarding its natural history, is currently scarce, it has been postulated that these patients would need total body cooling to reflect cold urticaria symptoms. ${ }^{1}$ A previous report also suggested a higher incidence of systemic reactions in these patients (10 patients with atypical ACU were analyzed, 6 presented with type II reactions and 2 with type III reactions). ${ }^{10}$ However, in our study, no statistically significant relationship was found between patients with atypical ACU and symptom severity (21 atypical cases; 11 type II and 1 type III). Nevertheless, a shorter duration of disease and lower doses of antihistamines required for achieving disease control were found in patients with atypical ACU. In addition, it seems that atypical cases have a shorter duration of cold urticaria symptoms regardless of the age of disease onset (eg, in our series, "classic" [ice cube positive] ACU in children lasted longer [7.6 years] compared to adults [ 5.2 years]). Therefore, clinicians should be aware that there is a subgroup of patients who develops cold induced urticaria or angioedema with atypical responses to CST, and although these patients should be followed and treated in a manner similar to classic cases, a more manageable disease would be expected.

Regarding atopy, a previous study suggested that patients with ACU who also present atopic features could have an increased severity of ACU symptoms. ${ }^{12}$ However, our results do not corroborate this finding, and it seems that clinical features of the disease, such as the age at symptom onset, the presence of atypical ACU, or the symptoms severity are independent of the presence of atopy.
The recommended first-line therapy for the management of patients with ACU is based on avoiding triggering factors, along with the continuous treatment with nonsedating $\mathrm{H}_{1}$-antihistamines (evidence level A). ${ }^{2,4,5,13}$ However, reduction of urticarial symptoms in many patients requires antihistamines at high doses, up to 4 times the standard dose. ${ }^{4,5,13-15}$ In our series, $85 \%$ of the patients who were treated with antihistamines showed significant clinical improvement. Rates of satisfactory clinical response with antihistamines ranged from 60\% to $70 \%$ in previous series. ${ }^{3,11,16}$ In contrast to cyclosporine, omalizumab was found to be an effective therapeutic alternative for patients with refractory ACU. ${ }^{4,17,18}$ Importantly, the presence of other family members with similar symptoms should always be ruled out in patients with ACU, because specific treatments (eg, anti-interleukin-1) have demonstrated effectiveness in the management of familial cold-induced urticaria syndromes. ${ }^{19-21}$ Moreover, regardless of the prescribed treatment, self-limited evolution can be seen in some patients with ACU. In the present study, almost 26\% of the patients experienced complete symptom resolution (and therefore no need for treatment) during the surveillance period, with a mean disease duration until resolution of 5.1 years. In addition, there was a resolution rate of $45 \%$ within the first 10 years. Rates of full recovery of symptoms among similar studies vary between $18 \%$ and 29\%, with variable durations of follow-up. ${ }^{9,12,16,22}$

In summary, although ACU represents a chronic disease and patients can occasionally experience life-threatening events, most subjects who receive treatment can achieve a daily life without interferences. Different phenotypes of patients with ACU are identified in routine clinical practice, and the knowledge of their clinical characteristics would improve their management. Some clinical features, such as the age at disease onset and an accurate severity assessment at baseline evaluation, may be helpful to predict disease evolution, allowing clinicians to define a proper therapeutic strategy.

\section{REFERENCES}

1. Wanderer AA. Cold urticaria syndromes: historical background, diagnostic classification, clinical and laboratory characteristics, pathogenesis, and management. J Allergy Clin Immunol. 1990; 85:965-981.

2. Siebenhaar F, Weller K, Mlynek A, et al. Acquired cold urticaria: clinical picture and update on diagnosis and treatment. Clin Exp Dermatol. 2007;32:241-245.

3. Alangari AA, Twarog FJ, Shih MC, et al. Clinical features and anaphylaxis in children with cold urticaria. Pediatrics. 2004;113: e313-e317.

4. Magerl M, Altrichter S, Borzova E, et al. The definition, diagnostic testing and management of chronic inducible urticarias - The 
EAACI/GA(2) LEN/EDF/UNEV consensus recommendations 2016 update and revision. Allergy. 2016;71:780-802.

5. Martinez-Escala ME, Curto-Barredo L, Carnero L, et al. Temperature thresholds in assessment of the clinical course of acquired cold contact urticaria: a prospective observational one-year study. Acta Derm Venereol. 2015;95:278-282.

6. Siebenhaar F, Staubach P, Metz M, et al. Peltier effect-based temperature challenge: an improved method for diagnosing cold urticaria. J Allergy Clin Immunol. 2004; 114:1224-1225.

7. Młynek A, Magerl M, Siebenhaar F, et al. Results and relevance of critical temperature threshold testing in patients with acquired cold urticaria. Br J Dermatol. 2010; 162:198-200.

8. Giménez-Arnau A, Ali Al-Haqan E, Sacrista M, et al. Ultra-freeze induced cold contact wheals during cryosurgery: an uncommon subset of acquired cold contact urticaria. Eur J Dermatol. 2013;23:694-699.

9. Neittaanmäki H. Cold urticaria. Clinical findings in 220 patients. J Am Acad Dermatol. 1985;13:636-644.

10. Wanderer AA, Grandel KE, Wasserman SI, et al. Clinical characteristics of cold-induced systemic reactions in acquired cold urticaria syndromes: recommendations for prevention of this complication and a proposal for a diagnostic classification of cold urticaria. J Allergy Clin Immunol. 1986;78:417-423.

11. Santaolalla Montoya M, Martínez Molero MI, Santaolalla San Juana F, et al. Cold urticaria: review of 12 cases. Allergol Immunopathol (Madr). 2002;30:259-262.

12. Katsarou-Katsari A, Makris M, Lagogianni E, et al. Clinical features and natural history of acquired cold urticaria in a tertiary referral hospital: a 10-year prospective study. J Eur Acad Dermatol Venereol. 2008;22:1405-1411.
13. Zuberbier $T$, Aberer $W$, Asero $R$, et al. The EAACI/GA(2) LEN/EDF/WAO Guideline for the definition, classification, diagnosis, and management of urticaria: the 2013 revision and update. Allergy. 2014;69:868-887.

14. Abajian M, Curto-Barredo L, Krause K, et al. Rupatadine 20 mg and $40 \mathrm{mg}$ are effective in reducing the symptoms of chronic cold urticaria. Acta Derm Venereol. 2016;96:56-59.

15. Magerl M, Pisarevskaja D, Staubach $P$, et al. Critical temperature threshold measurement for cold urticaria: a randomized controlled trial of $\mathrm{H}(1)$-antihistamine dose escalation. $\mathrm{Br} J$ Dermatol. 2012;166:1095-1099.

16. Buss YL, Sticherling M. Cold urticaria; disease course and outcome- an investigation of 85 patients before and after therapy. Br J Dermatol. 2005;153:440-441.

17. Le Moing A, Bécourt C, Pape E, et al. Effective treatment of idiopathic chronic cold urticaria with omalizumab: report of 3 cases. J Am Acad Dermatol. 2013;69:e99-e101.

18. Sussman G, Hébert J, Barron C, et al. Real-life experiences with omalizumab for the treatment of chronic urticaria. Ann Allergy Asthma Immunol. 2014;112:170-174.

19. Wanderer AA, Hoffman HM. The spectrum of acquired and familial cold-induced urticaria/urticaria-like syndromes. Immunol Allergy Clin North Am. 2004;24:259-286.

20. Lachmann HJ, Kone-Paut I, Kuemmerle-Deschner JB, et al. Use of canakinumab in the cryopyrin-associated periodic syndrome. N Engl J Med. 2009;360:2416-2425.

21. Hoffman HM, Throne ML, Amar NJ, et al. Efficacy and safety of rilonacept (interleukin-1 Trap) in patients with cryopyrinassociated periodic syndromes: results from two sequential placebo-controlled studies. Arthritis Rheum. 2008;58:2443-2452.

22. Van der Valk PG, Moret G, Kiemeney LA. The natural history of chronic urticaria and angioedema in patients visiting a tertiary referral centre. Br J Dermatol. 2002;146:110-113. 


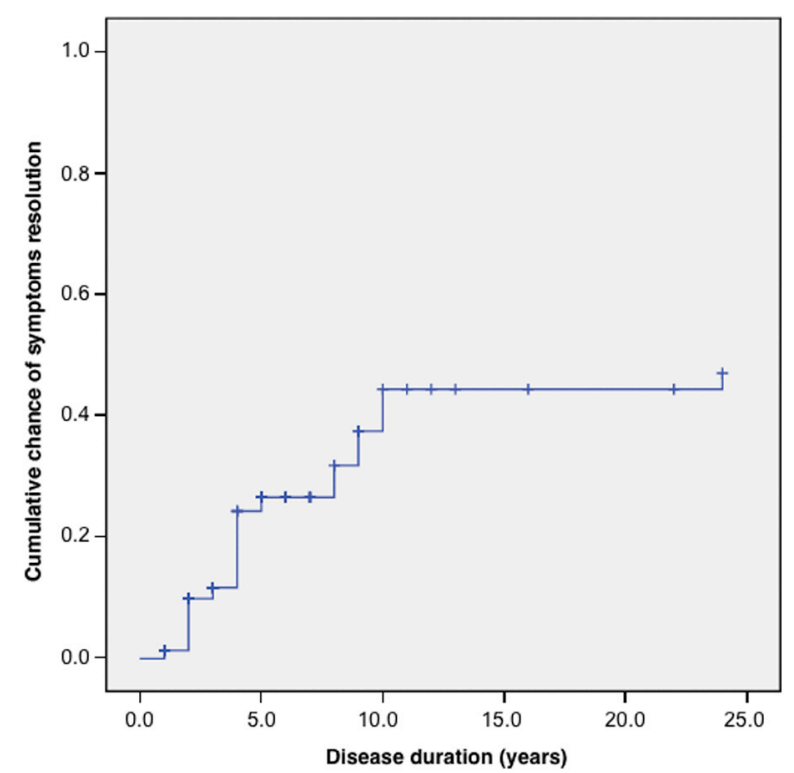

Supplementary Fig 1. Kaplan-Meier analysis showing the proportion of cases with symptom resolution at the different time points of disease evolution. 

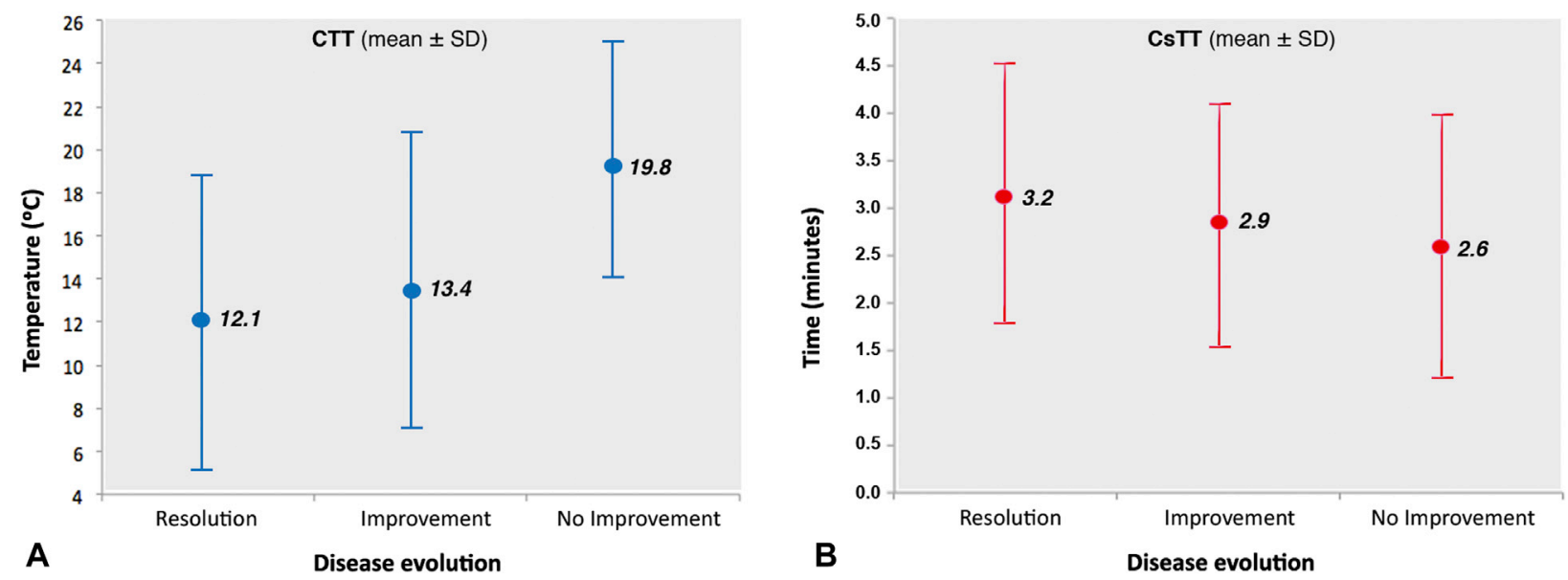

Supplementary Fig 2. A, Critical temperature threshold (CTT) and (B) cold stimulation time threshold (CsTT) values by categories of disease evolution ( $P=.03$ and $P=.54$; respectively). 